\title{
Erratum to: X-ray Lines in Binary Star Systems and the 3.55-keV Anomaly
}

\author{
V. V. Burdyuzha* \\ Astrospace Center, Lebedev Physical Institute, Russian Academy of Sciences, Moscow, 117997 Russia \\ *e-mail: burdyuzh@asc.rssi.ru \\ Received October 17, 2019
}

DOI: $10.1134 / \mathrm{S} 1063776119090152$

In Abstract, in the third sentence, the value of magnetic field should read $6 \times 10^{12} \mathrm{G}$.

The original article can be found online at

https://doi.org/10.1134/S1063776119060116 